\title{
A fresh look at desalination
}

\author{
Results from new artificial water channels for desalination show the importance of exploring alternative solutions \\ to simply optimizing existing technologies.
}

$\mathrm{C}$ lean water for drinking, for agriculture and for sanitation has become an increasingly precious resource, primarily as a consequence of climatic changes and of overpopulation. The challenge has to be tackled from different fronts, including improved infrastructures for efficient distribution as well as adequate behaviour to avoid wastes. Technology also plays an essential role, for example clean water can be obtained by removing salt from seawater or brackish water.

Reverse osmosis is perhaps the most popular desalination process. Salty water gets pushed through a porous membrane at high pressure and the membrane captures salt while letting water permeate through. In simple terms, the efficiency of a membrane can be measured by comparing how much water can permeate and how much salt can be rejected. Unfortunately, permeability and selectivity tend to compete against each other. The best results can be obtained with thin-film composite (TFC) polymer membranes, which are in fact used commercially, and despite efforts to look at other materials, so far no definitive improvement has been demonstrated.

In an Article published in this issue, Woochul Song et al. report promising results

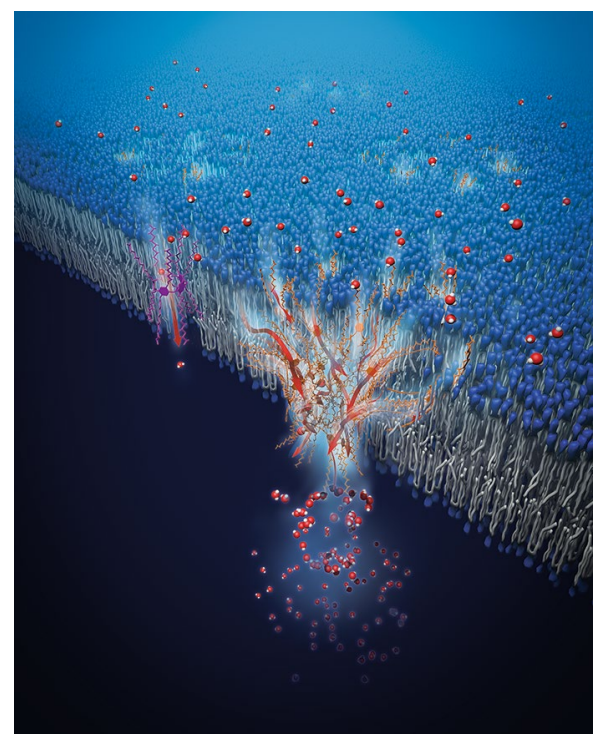

Artistic impression of water flowing through the artificial water channels studied by Song et al. Credit: Erik Zumalt, The University of Texas at Austin

using membranes based on artificial water channels. Membranes mimicking aquaporins have been investigated before. The new aspect of this work is that the water does not flow through static channels created in the membranes. Rather, the nanoarchitecture that forms the membranes results in the formation of a network of water wires that allows a permeability similar to that of natural aquaporins and a very high salt selectivity, enough to predict better performance than TFCs, once scaled up.

Of course, scaling up may end up being a big challenge. But the essential aspect of the work is that it provides a clear example of a case in which - when a technology reaches its limits - the best way forward is to go back to the drawing board and explore issues from a fundamental perspective. We have reason to think that this type of approach is becoming more urgent in more branches of nanotechnology, including, for example, medical and energy applications. In this specific case, whether the membranes investigated will themselves end up in commercial applications is secondary to the potential inspiration for new ways to design membranes that the results may inspire.

Published online: 10 January 2020 https://doi.org/10.1038/s41565-019-0627-3

\section{Good luck, Teri Odom}

\section{We reflect on the major role played by Nano Letters in nanoscience and nanotechnology and celebrate the appointment of its new editor-in-chief.}

A s one of the first ever journals launched with the word 'nano' in the title, Nano Letters has been a pioneer publication in nanoscience and nanotechnology. The first issue editorial of 28 November 2000, signed by Paul Alivisatos, explained that bottom-up and top-down approaches were converging at the nanometre scale and would, at some point, allow the fabrication of materials with complete control of composition and therefore functions at all scales. A science that is not chemistry or physics or biology required a truly interdisciplinary journal. And within interdisciplinarity lies the journal's success.

Understanding nanoscale systems requires that each discipline contributes with its own perspective and investigative tools to create something new that ultimately transcends traditional fields. Furthermore, findings that elucidate effects that at first sight seem specific to a certain nanoscale system may well be of interest to a much wider audience, as these interactions operate on and are relevant to that same length scale. As such, publishing nanoscale research in specialized journals may not reach the intended audience; or, better put, may not reach all those researchers that could potentially benefit from it. Kudos, then, to the American Chemical Society for betting on this vision and to Alivisatos and Charles Lieber who have been co-editing the journal and led the way.

Over the years, there has been a consolidation of this way of thinking. Nanoscience and nanotechnology research has exploded and many more journals have been launched (including Nature Nanotechnology back in 2006). Moreover, we have also seen an evolution of the composition of individual research groups. In more and more cases - where funding allows - principal investigators are trying to combine chemists, physicists, electrical engineers, biologists and so on in their own lab to make it interdisciplinary to start with. This way of working naturally fosters collaboration and consolidates further the foundational elements of nanoscience and nanotechnology research. From this perspective, we find the choice of Teri Odom particularly appropriate, as her group is also set up in this way and she is therefore wellplaced to interpret the evolution of the field in the near future.

We look forward to seeing her vision reflected in the journal, and to co-operating with Nano Letters in promoting nanoscience and nanotechnology research towards the solution of societal challenges.

Published online: 10 January 2020 https://doi.org/10.1038/s41565-019-0628-2 